\title{
Hardware in the loop test-rig for identification and control application on high speed pantographs
}

\author{
A. Collina ${ }^{\mathrm{a}}$, A. Facchinetti ${ }^{\mathrm{a}}$, F. Fossati $^{\mathrm{b}, *}$ and F. Resta ${ }^{\mathrm{a}}$ \\ ${ }^{a}$ Dipartimento di Meccanica, Politecnico di Milano, Milano, Italy \\ ${ }^{\mathrm{b}}$ Dipartimento di Ingegneria Industriale e Meccanica, Universitá di Catania, Catania, Italy
}

\section{Caro Bruno,}

ti abbiamo incontrato per la prima volta, ad un congresso, da giovanissimi ricercatori, insicuri da un punto di vista scientifico e intimoriti al pensiero della tua presenza in sala. Da subito ci hai mostrato una grande umanit á oltre al tuo desiderio di insegnare anziché di mostrare quanto sapevi.

Ci siamo successivamente visti in numerose occasioni. Nonostante i problemi personali e gli attriti tra colleghi che spesso sono presenti nel nostro ambiente, tu avevi sempre il tempo per chiederci come procedevano la carriera e le ricerche a cui ci dedicavamo.

Quando abbiamo ricevuto l'invito di Luigi a mandare un lavoro per un numero commemorativo, abbiamo avuto il dubbio se vi fossero ricerche che saremmo stati fieri di presentarti. Pur non trovando nulla di adeguato, abbiamo selezionato un tema su cui lavoriamo da tempo che coniuga l'impostazione di carattere applicativo che ti contraddistingueva, all'utilizzo delle metodologie di identificazione dei parametri e di controllo attivo di cui sei sempre stato un importante punto di riferimento per tutti noi e per tutta la comunitá scientifica. Speriamo che ció ti sia gradito.

\begin{abstract}
Trains current collection for traction motors is obtained by means of a sliding contact between the overhead line (OHL) and the collector strips mounted on the pantograph head. The normal force by which the collector presses against the contact wire ensures the contact pressure for the electrical contact. As the train speed increases, the variation of contact force between pantograph and catenary increases, and the pantograph-OHL dynamic interaction becomes greater. This condition causes excessive mechanical wear and contact wire uplift (for high values of contact forces), and leads to high percentage of contact loss, arcing and electrically related wear.

The topic of actively controlled pantograph is gaining more interest as a tool to increase the performance of the current collection at high speed. In the last few years, it appears possible to transfer the knowledge based on numerical experiments, to the real operating condition. An important step in this direction is the set up of a laboratory hardware in the loop test-rig in which the control strategies and the actuation can be tested, before tests performing in real life conditions, in order to demonstrate their actual feasibility. The present paper describes an hardware in the loop (HIL) test-rig developed by the authors, which allows to reproduce the dynamical interaction between overhead lines and pantograph in high speed railways. Using the described laboratory set-up, experimental investigation on the problems related with pantograph-OHL interaction can be performed, very similarly to the real life operating conditions, with the advantage of varying test parameters and conditions easily.
\end{abstract}

\footnotetext{
${ }^{*}$ Corresponding author. E-mail: fabio.fossati@ @mec.polim.it.
} 


\section{Introduction}

Pantograph-catenary dynamic interaction has been the subject of many researches $[3,6,12,14,16]$, with particular reference to high speed applications, with the main objective of increasing the quality of current collection through the reduction of contact losses and, more in general, of the variations of the contact force.

Active control application has been issued as a means to improve pantograph performance, overcoming the limitations which are encountered, when train speed approaches $300 \mathrm{~km} / \mathrm{h}$. A pantograph active control strategy is foreseen to have a positive impact also on more general aspects of pantograph-OHL interaction:

- it would enable increase of performance meaning higher speed, still having satisfactory current collection quality and lower level of wear both on the collector strips and on the contact wire;

- interoperability is also enhanced, allowing, in principle, to run with the same pantograph under catenaries with different mechanical features, with a minimum loss of performance [16];

- since an active control of the pantograph implies the knowledge and/or the estimation of the contact force, this information could also be used as a diagnostic tool, enabling to detect the condition of the OHL, cooperating with the activity of specialised diagnostic trains, which cannot run every day on the whole network.

Up to now the experience of actively controlled pantograph has been carried out in laboratory [15] and sporadically with full scale tests $[2,4]$. The main challenge is to demonstrate the performance of the active control applied to the pantograph, in a safe and controlled environment, using the same devices that will be installed on the pantograph and getting as close as possible to the operating conditions.

This would help to step forward the in-line test of active controlled pantograph.

The starting point is a detailed mathematical model of pantograph-OHL dynamic interaction [5], into which the control strategy and the actuator dynamics can be included [9].

In previously published papers $[9,10]$, authors investigated the possibility to improve the current collection quality by means of contact force control strategy, aiming at the reduction of the contact force dynamic variation. Active control action is superimposed to the already existing preload acting on the pantograph.

In order to build up a control system, the contact force is therefore used as a controlled variable and the problem of its measurements plays a dominant role.

The main problem is related to the lack of information on the catenary state and to the difficulty related to a contact force direct measure. Due to these reasons, in the development of an active control system, only the pantograph motion is considered measured data available, while the contact forces is supposed to be estimated via the Extended Kalman Filter (EKF) technique [9]. Nevertheless, before control strategy and contact force estimation can be implemented for in-line tests, it is necessary to experimentally verify their feasibility.

Contact force estimation procedure effectiveness has been previously checked using experimental measurements carried out during in-line tests. The use of the measured displacements as observable variables, together with a reference model, allows the contact force estimation in a quite satisfactory way, as stated by comparing it with the measured one. The consistency of this approach have been tested with measured values [7].

Design and realisation of a laboratory hardware in the loop (HIL) test-rig is the main subject of the research currently in progress: this device allows to verify, both control strategy and contact force estimation procedure using a real pantograph, in similar conditions to real life line case.

The HIL test-rig described in the following consists of an hardware in the loop simulator, similar to what proposed in [18] and it is constituted by a controlled electro-hydraulic actuator which is coupled to the real pantograph. Actuator input is the reference position of $\mathrm{OHL}$ in correspondence of the pantograph collector, obtained through a numerical model of a catenary travelled by a vertical force (the contact force). OHL dynamics simulation runs on a real-time CPU, accepting as input the contact force measured between the pantograph and the hydraulic actuator. In this way an hybrid simulation is carried out, where OHL is numerically simulated and a real pantograph is actually working.

In the paper some preliminary obtained results together with some results concerning the EKF application for contact force estimation (instead of measurements) are shown too.

As a complementary research activity, the implementation of the actuation proposed in [17] has been performed in a dedicated laboratory bench, where a single collector is controlled by means of a DC motor.

Future actions will be the installation of the complete pantograph equipped with an active controller, on the HIL simulator. 


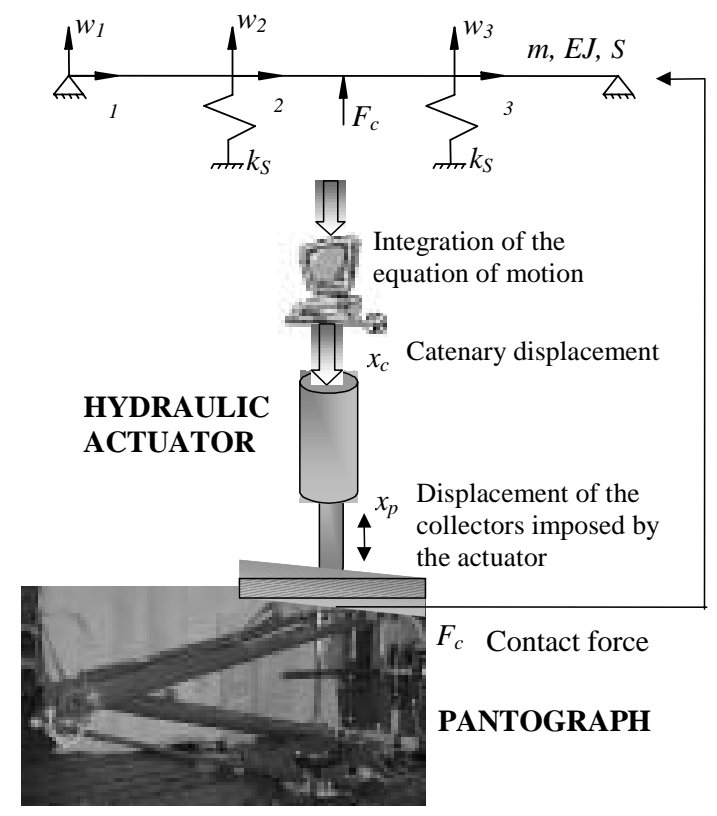

Fig. 1. The Hardware in The loop HIL test-rig scheme.

\section{The hardware in the loop test-rig}

In order to analyse the dynamical behaviour of the pantograph and catenary system, it is necessary to reproduce the interaction between the two sub systems. For this purpose a HIL test-rig has to take into account both the pantograph dynamics due to the catenary motion and the catenary response due to the contact force.

The idea [18] consists in installing a pantograph in the laboratory and coupling the collectors with a displacement controlled hydraulic cylinder.

The control system imposes to the hydraulic cylinder a motion law derived by a numerical model of the catenary excited by the contact forces, actually measured in the HIL test-rig.

In this way, the pantograph is moved by the actuator which imposes the displacements of the collectors: the contact force between the pantograph and the hydraulic actuator is measured and fed back in the catenary model.

An hardware in the loop device has been in this way realised: the real pantograph dynamics is coupled to a hydraulic cylinder controlled by a PC (Fig. 1). A simplified numerical model of the catenary, forced by passing of the directly measured contact force, allows us to evaluate the time history of the displacements of the point of the line in contact with the collector. In real time, this time history is imposed as references to the control system of the actuator.

The feasibility of the proposed approach was previously checked simulating the complete experimental set-up by means of a numerical model, realised with Simulink. The various elements were modelled as follows:

- the catenary was reproduced by a simplified model, described in the following section;

- the real pantograph was substituted with a lumped parameters model;

- the hydraulic actuator was represented by means of its transfer function between required and actual position.

After the numerical validation of the HIL test-rig design [13], a pantograph and a hydraulic actuator have been installed in the laboratory and the catenary model has been implemented into a acquisition and control board (Dspace 1102 based on DSP Texas TMS320C31), which is programmable using an high level interface in SIMULINK/MATLAB ${ }^{\circledR}$ environment. The board interacts with the bench through an analog input, represented by the measure of the actual contact force between the hydraulic actuator and the pantograph, and an analog output, consisting in the reference position required to the actuator.

Figure 2 shows a picture of the realised HIL test-rig. 


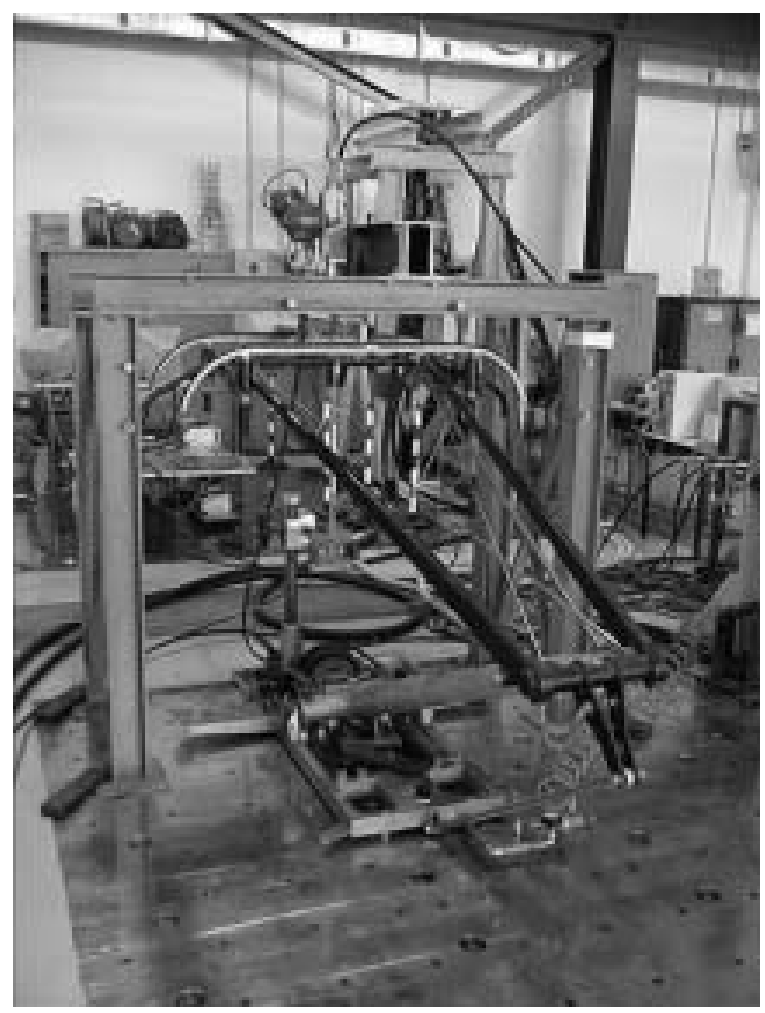

Fig. 2. The HIL test-rig.

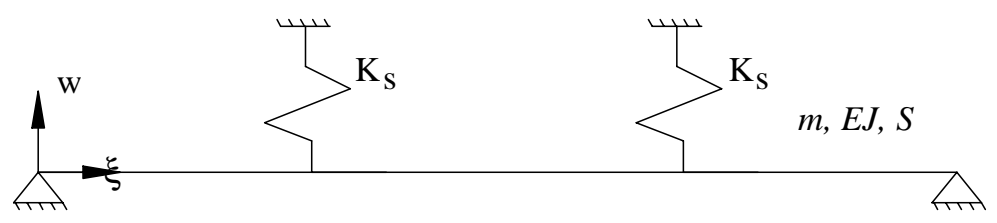

Fig. 3. Three span model of the catenary.

\subsection{The numerical model of the catenary}

In order to perform a real time execution of the HIL device, a fast integration of the motion equations is required and, therefore, a simplified numerical model of the catenary has to be adopted, since more complex models (e.g. F.E.M based models) involve an unacceptable computational effort.

Authors propose a simplified numerical model, where the catenary is represented as a single tensioned wire with $m, E J$ and $S$ representative of the whole OHL, while the suspension sets are modelled by means of equivalent stiffness $K_{S}$, as shown in Fig. 3.

The catenary is modelled by means of a modal approach, while the presence of droppers is neglected in order to limit the computation time needed for the solution of the model equation and for the hydraulic cylinder real time control.

Considering a limited number of vibration modes, it is possible to reproduce the most important aspects of catenary dynamic behaviour, at least with respect to the harmonic components of the contact forces related with span passing.

Using a modal approach, the vertical motion of the contact wire can be written as follows:

$$
w(\xi, t)=\sum_{k=1}^{\infty} \Phi_{k}(\xi) q_{k}(t)
$$



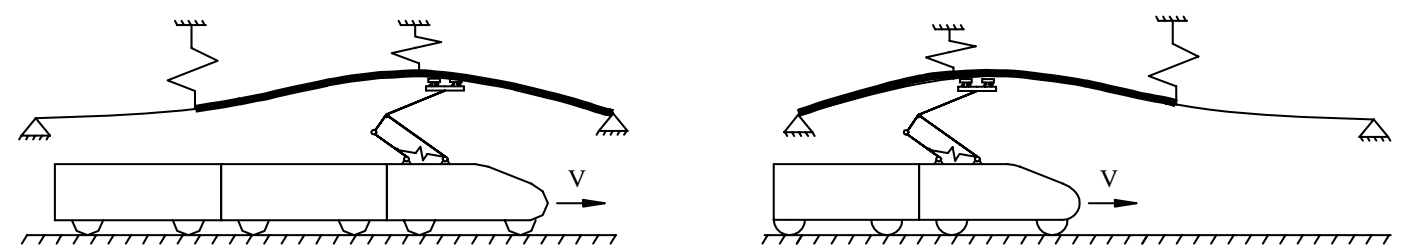

Fig. 4. Transition between two subsequent spans.

where $w(\xi, t)$ is the vertical displacement, which depends on the longitudinal position $\xi$ and on time $t$, $\Phi_{k}(\xi)$ is the $k^{\text {th }}$ modal shape, depending only on the longitudinal position, and $q k(t)$ is the $k^{\text {th }}$ modal coordinate, depending only on time.

Assuming to consider only a limited number of modes $\Phi(\xi)$, the vertical displacement of the contact point can be approximated to:

$$
w_{c}\left(\xi_{C}, t\right)=\underline{\Phi}\left(\xi_{C}\right)^{T} \underline{q}(t)
$$

where:

$$
\begin{aligned}
& \underline{\Phi}(\xi)=\left[\begin{array}{lll}
\Phi_{1}(\xi) & \Phi_{2}(\xi) \ldots \Phi_{k}(\xi) \ldots \Phi_{n}(\xi)
\end{array}\right]^{T} \\
& \underline{q}(t)=\left[\begin{array}{lll}
q_{1}(t) & q_{2}(t) \ldots q_{k}(t) \ldots q_{n}(t)
\end{array}\right]^{T}
\end{aligned}
$$

and $\xi_{C}$ is the contact point position along the line, defined in order to place the pantograph always interacting with the central span, as better described in the following.

The equation of motion are obtained by means of a Lagrangian approach, and have the following form:

$$
[M] \underline{\ddot{q}}+[C] \underline{\dot{q}}+[K] \underline{q}=\underline{Q}(t)
$$

where $[M],[C],[K]$ are the mass, damping and stiffness matrices and $Q$ is the Lagrangian generalised contact force vector, which contains pantograph contact force contributes.

The modal damping matrix $[C]$ has been evaluated in order to reproduce the system damping modal ratios in the frequency range considered by the modal superposition.

With reference to the requirement of real-time execution, it was decided to not reproduce the whole OHL, but to limit the analysis of the OHL in the neighbourhood of the region interested by pantograph interaction phenomena.

The numerical model considers the schematisation of only three spans; the pantograph interacts always with the central span, which is the only one presenting boundary conditions like those of a real span. Every time pantograph reaches the end of the central span, it is virtually put back to the beginning of the same span by means of a suitable algorithm: $\xi_{C}$ is imposed to be equal to $L_{0}$ value and the integration process proceeds with new initial conditions, corresponding to the pantograph exit from the central span:

$$
\xi_{C}=V t-(n-1) L_{0}
$$

where $V$ is the running speed, $L_{0}$ the span length and $n$ the number of covered spans.

The deflection shapes of the second and third span are reported to the first and second span. The modal coordinates are then calculated by means of a least square minimisation, imposing the so defined deformed configuration, and they are used as initial conditions for the following integration steps (Fig. 4).

This approach allows, with a very low computational effort, the interaction between pantograph and the OHL simulation avoiding the use of a model with a number of spans depending on the simulated case.

Moreover this technique allows a good compromise between low computational effort, due to real time simulation requirements, and the possibility of reproducing the over-whole catenary + pantograph system frequency range related to span passage dynamics. 


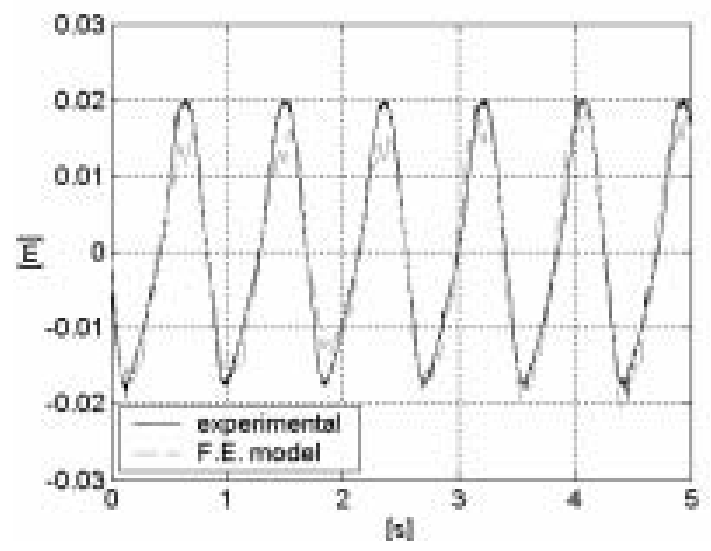

Fig. 5. Collectors displacement.

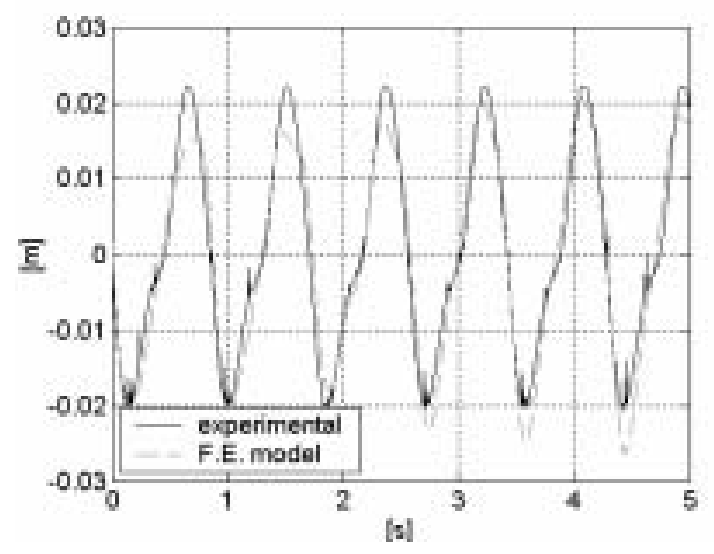

Fig. 6. Articulated frame displacement.

\section{Dynamic response of the hardware in the loop test-rig}

Using the described laboratory device, different tests were carried out, referring to a Dornier DSA350 pantograph.

The considered OHL is composed of a single messenger wire and a single contact wire. In the simplified schematisation, the single tensioned wire present the characteristics obtained as a superposition of messanger and contact wires (tension $16+20 \mathrm{kN}$, mass per unit length $1.07+1.35 \mathrm{~kg} / \mathrm{m}$ ) data.

The considered pantograph has two collector heads. Nevertheless, the connection between the hydraulic actuator and the two collectors have been realised with a rigid rod, which actually constrains the displacements of the two collectors to be the same.

Figures 5-8 show some meaningful quantities, measured during the experimental tests on the HIL test-rig, in comparison with the same results obtained by means of the complete numerical model developed during previous research: this is based on a F.E.M. schematisation of the OHL and on a pantograph lumped parameters model and it has been extensively validated by means of many tests performed in real life operation conditions [5]. The numerical model has then been used as reference, in order to verify the capability of the laboratory device to reproduce the dynamic behaviour of the real overhead system.

Figure 5 shows the time histories of the measured collectors displacement (only the dynamic component is considered) against the one obtained with the numerical model, while Fig. 6 reports the same quantities with respect to the articulated frame. 


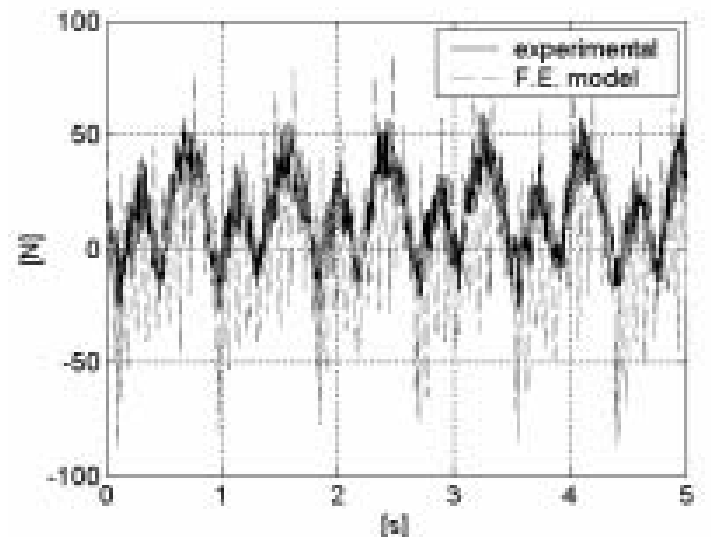

Fig. 7. Contact force variation.

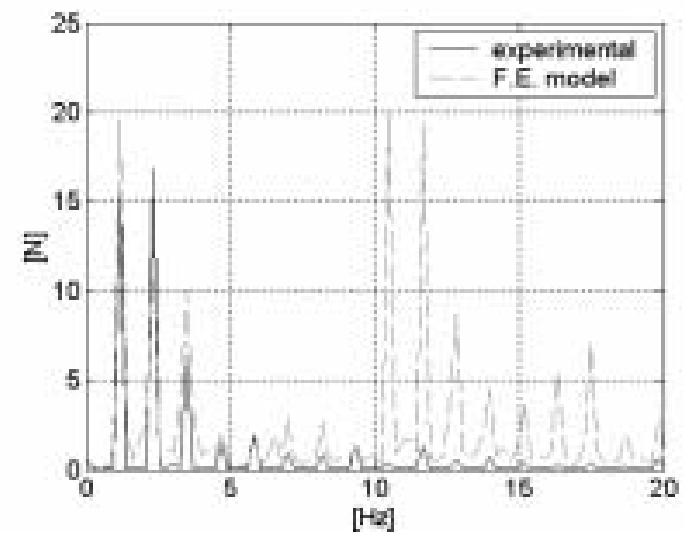

Fig. 8. Contact force spectrum.

It can be noticed a satisfactory agreement between the trends provided by the complete F.E. numerical model and those obtained experimentally with the HIL test-rig

Finally, Fig. 7 shows the comparison of the time histories of measured and simulated contact force.

In this case, greater differences arise as a consequence of droppers contribution, which is accounted for in the F.E. numerical model but neglected in the simplified model adopted for driving the hydraulic actuator.

Considering the contact force spectrum (Fig. 8), a good agreement can be observed in the amplitude of harmonic contribution up to $5 \mathrm{~Hz}$, while substantial differences are present in the range of frequencies higher than $10 \mathrm{~Hz}$, which corresponds to the frequency of droppers passing, for the considered running speed.

\section{Some applications of the hil test-rig}

The realised HIL test-rig allows to study the dynamic behaviour of the OHL-pantograph system, performing a sensitivity analysis of main design parameters; and represents a useful mean to simulate design changes before full scale tests.

On the other hand, some research applications can be applied and verified effectively, by the direct use of the HIL lab-rig.

In the following some meaningful studies, performed and in progress, are presented, which rely on the developed HIL test-rig: 


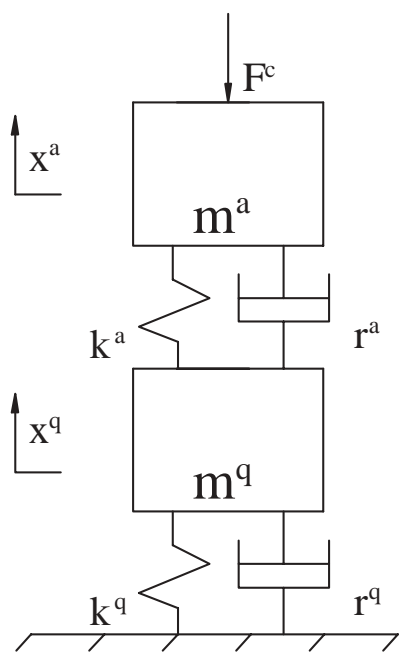

Fig. 9. Lumped parameters reference model of the pantograph.

- contact force estimation procedure;

- diagnostic tools;

- active control of the pantograph.

\subsection{Contact force estimation}

The first issued topic has been the estimation of the contact forces between the pantograph and the OHL. In fact, the knowledge of the contact force represents the basis for the other proposed topics, since it is fundamental in order to assess the state of the OHL and represents the feedback for the active control system. Nonetheless, it is well known that the direct measurement of the contact force is a challenging task [4] and therefore an alternative way to accomplish the task is sought for.

To this end a contact force estimation procedure based on the Extended Kalman Filter algorithm has been developed [9].

The extended Kalman Filter is a recursive algorithm that allows to have an estimation of the extended state vector using experimental measures of the system dynamics $y(t)$ (named observations). System state estimation of the effective extended state $z(t)$ (and of the parameters $p$ together) is performed in such a way to minimise the state estimation error variance.

The reference model of the EKF is a lumped parameter model with 2 D.O.F. (the collectors and articulated frame displacements) (Fig. 9).

The motion of the pantograph is considered as the observable state, while the contact force is considered as an extension of the state vector:

$$
\underline{z}=\left[x_{a} x_{q} \dot{x}_{a} \dot{x}_{q} F c\right]^{T}
$$

where $\underline{z}$ is the extended state vector, $x_{a}$ and $x_{q}$ are respectively the collectors and the articulated frame displacements and $F_{C}$ is the contact force.

Figure 10 summarises the contact force estimation procedure by means of the EKF technique.

Two versions, considering the observation of both displacements and velocities, or displacements only, have been developed, being the second one to be considered for the real application.

The estimation procedure was tested on the HIL test-rig. In the following, some of the obtained results are shown.

Figure 11 shows the time histories of the contact force measured with the load cell during the tests (experimental) and of the estimated contact force with running speed $250 \mathrm{~km} / \mathrm{h}$ and static load $120 \mathrm{~N}$. The estimation was carried out using as observations for the EKF the measured displacements and velocities of the collectors and of the articulated frame. The obtained results are extremely satisfactory. 


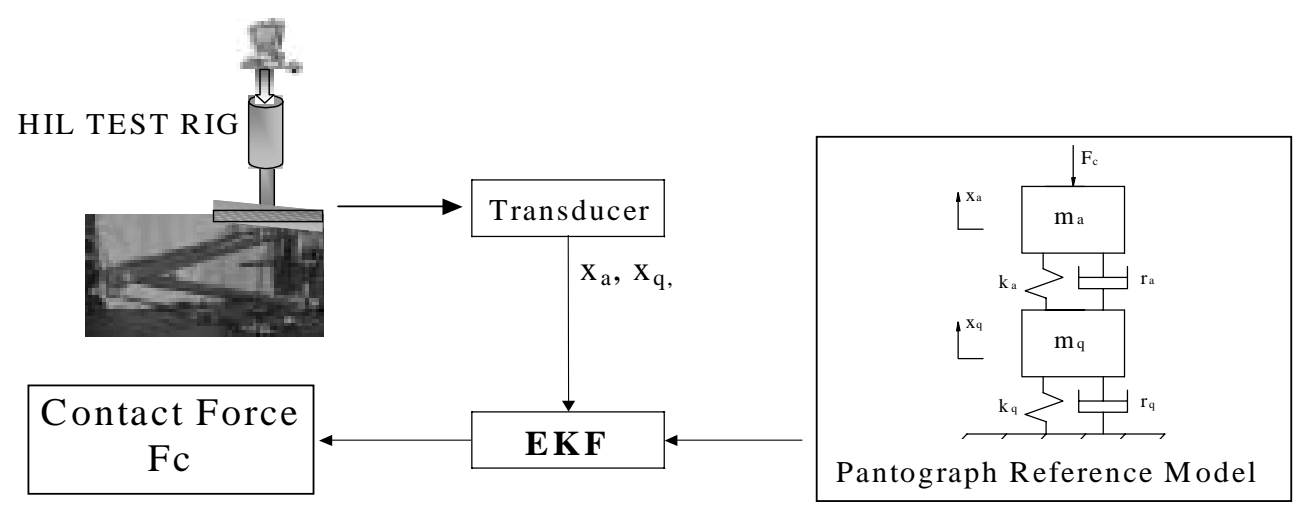

Fig. 10. Contact force estimation procedure.

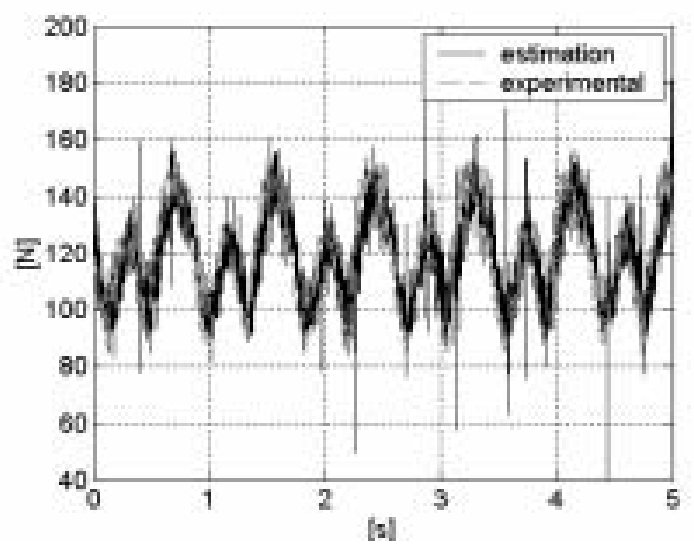

Fig. 11. Contact force estimation through the observation of both displacements and velocities of the pantograph .

Figure 12 reports the same comparison but in the case of the only displacements used as observation inputs to the filter.

In that case, even if the agreement is still good, a certain delay appears on the obtained estimation with respect to the measured contact force. This effect is due to the increased phase shift introduced by the filter as a consequence of the reduction of the number of observations. It is worth remarking that the observed delay doesn't affect significantly diagnostic applications, while more attention must be paid when the estimation is used for active control purposes.

\subsection{Diagnostic of the overhead line}

Based on the contact force estimation a diagnostic procedure has been developed [8] and applied to both numerically simulated defects and to experimental data referring to defect on a real OHL. The research activity demonstrated that the statistical analysis of the estimated contact force allows to identify the OHL failure presence and defect typology.

Different catenary defects can be modelled and included in the HIL rig in order to further investigate the proposed diagnostic tools capabilities and effectiveness.

\subsection{Active control}

In this section, a state-of-the-art of research on active controlled pantograph is briefly reported in order to clarify the activity about active control on the HIL test-rig. 


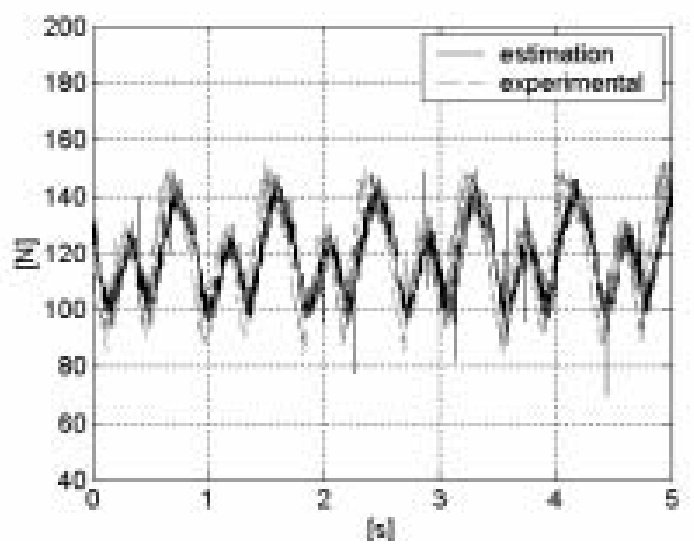

Fig. 12. Contact force estimation through the observation of the only displacements of the pantograph.

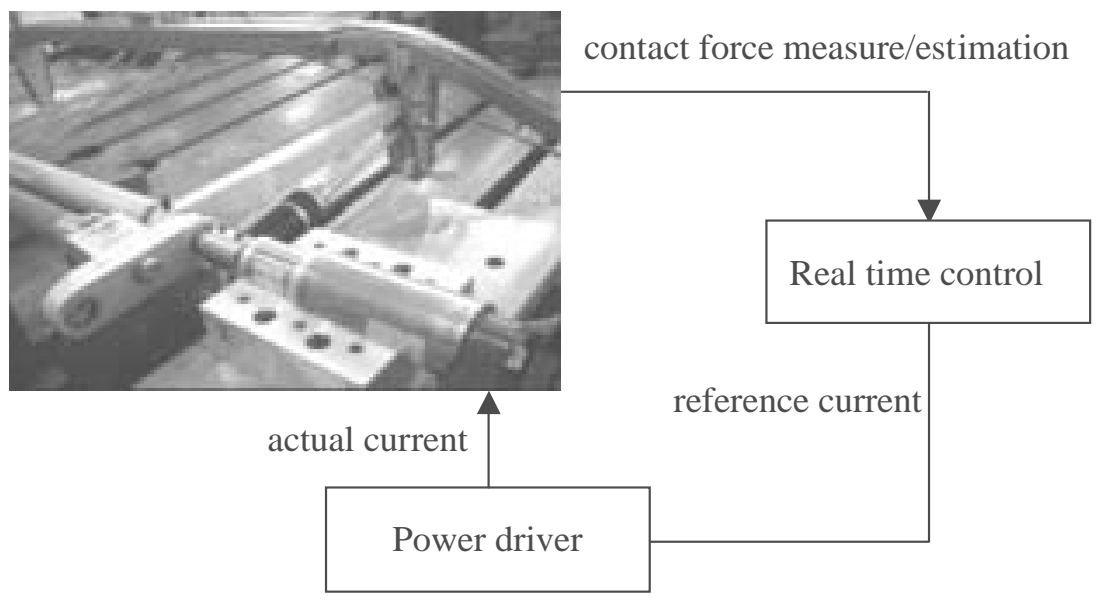

Fig. 13. Pantograph active control.

Two approaches can be applied when considering controlled pantographs [12,16]: to adopt a completely controlled pantograph, or adding the control actuators in parallel to the structure and the suspensions of the pantograph. Concerning the second approach, the actuators can be placed on the articulated frame, or in correspondence of the collector head suspension $[9,12]$.

The first configuration has the advantage that there're no strict limitation to the size, power, mass and kind of the actuator (pneumatic, hydraulic or electric). Nevertheless its effectiveness is limited to the low frequency range.

The second configuration chosen by the authors is more effective for the control of the medium frequency range but it imposes severe limitations to the size and mass of the actuators to be placed in correspondence of the collector head suspension. The activities related to the research have been developed through the following steps:

- preliminary assessment of the feasibility of the control configuration, by means of a simplified model of pantograph-catenary interaction, adopting a simple proportional control scheme, where the controlled variable is the contact force [9]. Aim of the simplified model is to roughly define the control system configuration;

- refinement of the results from the previous step, by means of a complete model of the pantograph-catenary dynamic interaction, considering ideal actuators;

- selection of a suitable actuator, and evaluation of its mounting on the real pantograph ATR95 developed by AnsaldoBreda for the ETR500 train set;

- modelling of the actuator, and implementation of the controlled actuator in the complete model for the pantograph-catenary dynamic interaction [17]; 

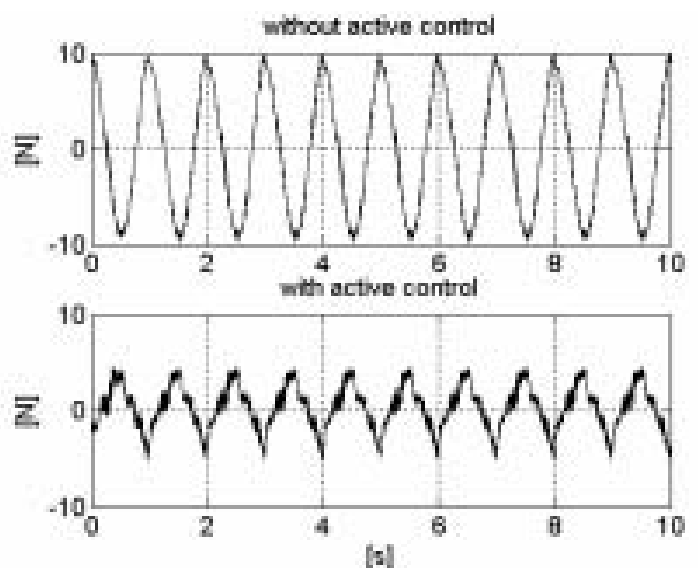

Fig. 14. Contact force variation.

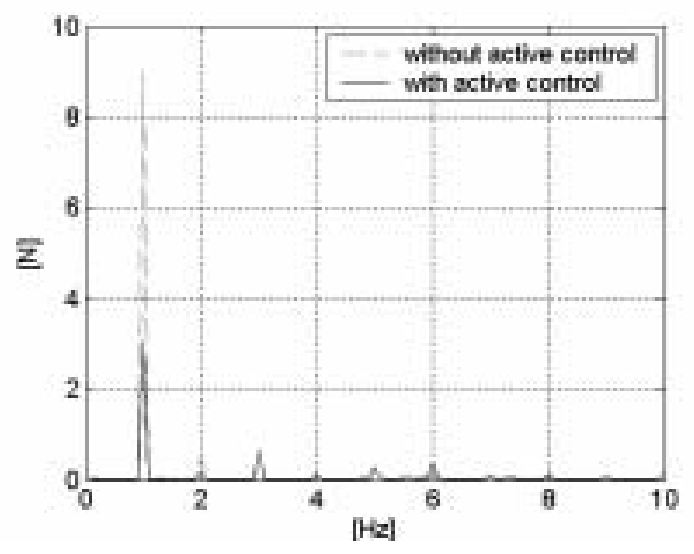

Fig. 15. Contact force spectrum.

- application of the active control to the extension of the travelling train speed, simulating an existing pantograph - catenary combination.

- realisation of a dedicated laboratory bench [1], where a single collector is controlled by a DC motor (Fig. 12), for a preliminary validation of control strategies.

More in details, the active control of the pantograph is realised by controlling the rotations of both collector head arms. This is obtained by means of two electric motors applied inside the joint between pan-head and each collector head arm (Fig. 13).

The actuator consists in a electromechanical system including a DC motor and a gearbox. A DC servomotors with relatively small power ratings characterised by extremely small time constants and the high torque-to-inertia ratio has been chosen. The armature -controlled DC motor is driven by an electronic controller operating in force control mode.

Figures 14 and 15 show an example of preliminary results obtained using the previously mentioned dedicated laboratory bench and considering a single collector, in terms of reduction of the contact force fluctuation achieved with the adoption of an active control.

The future planned action will be the installation of the complete pantograph with the active controller, on the HIL simulator. 


\section{Concluding remarks}

An HIL test-rig has been described, for the investigation of pantograph-OHL dynamic interaction. The HIL test-rig is composed by hydraulic cylinder which is controlled in order to reproduce the dynamic behaviour of the OHL, obtained by means of a simple numerical model, coupled to a real pantograph.

Some preliminary results have been shown, concerning the verification of the capability of the HIL test-rig to reproduce the dynamic behaviour of the real overhead system, by means of comparison with the results obtained through a validated numerical model of pantograph-OHL interaction, and a first application of the laboratory device for the testing of a contact force estimation procedure based on the Extended Kalman Filter algorithm. The developed HIL test-rig is currently able to reproduce the dynamic behaviour of the pantograph-OHL system, in the range of frequency related to the harmonics of the span passing frequencies, and it can therefore already be used for the development and verification of pantograph active control strategies.

Future developments involves the installation of the complete pantograph with the active controller on the HIL simulator, and the introduction of non linear droppers contribution in the numerical model adopted for driving the hydraulic actuator, in order to extend the range of reproducible frequency.

\section{References}

[1] F. Abbiati and S. Divina, Realizzazione sperimentale di un sistema di controllo attivo per un pantografo per treni ad alta velocith, Graduate thesis, Politecnico di Milano, 2002.

[2] B. Allotta, M. Papi, L. Pugi, P. Toni and A.G. Violi, Experimental campaign on a Servo-actuated pantograph, AIM ‘01, Como, Italy, July 2001.

[3] K.A. Althammer and W. Baldauf, Simulation of actively controlled pantographs in overhead line systems, WCRR '97, Florence, 16-19 Nov. 1997.

[4] K.A. Althammer and W. Baldauf, Considerations on high performance pantographs, WCRR '99, Tokio, Oct. 1999.

[5] S. Bruni and A. Collina, Numerical simulation of Pantograph-Overhead Equipment Interaction, Vehicle System Dynamics, Swets \& Zeitlinger Pub. Lisse, NE, 38(4) (October 2002), 261-292.

[6] A. Buter, E. Breitbach and H. Hanselka, Adaptive structure technology to reduce the dynamic interaction between current collector and aerial conduit, WCRR '97, Firenze, Italy, Vol. C, 1997, pp. 201-207.

[7] D. Carillo, A. Collina, A. Facchinetti, F. Fossati, M. Papi and F. Resta, Application of EKF to the estimation of contact forces in pantograph-catenary system, WCRR '03, Edinburgh, UK, Sept. 2003.

[8] A. Collina, F. Fossati and F. Resta, An innovative OHL diagnosis procedure based on the pantograph dynamics measurements, WCRR '01, Koln, Germany, Sept. 2001.

[9] G. Diana, S. Bruni, A. Collina, F.Fossati and F. Resta, High speed railways: pantograph and overhead line modelling and simulation, Proceedings of Comprail '98, Lisboa, in Computer in Railways VI, Wit Press, 2-4 September 1998, pp. 847-856.

[10] G. Diana, F. Fossati, F. Resta and A. Collina, Active control of high speed train pantograph, IIIrd Int. Conf. On Motion and Vibration Control, Chiba, Japan, Sept. 1996.

[11] G. Diana, F. Fossati and F. Resta, High speed railway: pantograph active control and overhead lines diagnostics, Vehicle System Dynamics, Swets \& Zeit Pub, Lisse, NE, Vol. 30, 1998

[12] G. Galeotti, M. Galanti, S. Magrini and P. Toni, Servo actuated railway pantograph for high-speed running with constant contact force, IMechE 1993, Vol. 207.

[13] F. Mignemi, Dispositivi per la simulazione del comportamento dinamico del sistema di captazione elettrica nei treni ad alta velocí, Graduate thesis, Universitá di Catania, 2002.

[14] D.N. O'Connor, S.D. Eppinger, W.P. Seering and D.N. Wormley, Active Control of a High-Speed Pantograph, ASME Jour. Of Dynamic Systems, Measurements and Control 119(1), (March 1997).

[15] M. Papi, M. Rinchi, A. Rindi and P. Toni, Preliminary field testing of a servo-actuated pantograph, Computer in Railways VI, WIT press, 1998, pp. 837-846.

[16] G. Poetsch, J. Evans, R. Meisinger, W. Kortum, W. Baldauf, A. Veitl and J. Wallaschek, Pantograph/Catenary Dynamics and Control, Vehicle System Dynamics 28(2/3) (1997), 159-195.

[17] F. Resta, A. Collina and F. Fossati, Actively controlled pantograph: an application, AIM '01, Como, Italy, July 2001.

[18] W. Zhang, G. Mei, X. Wu and Z. Shen, Hybrid Simulation of Dynamics for the Pantograph-Catenary, Vehicle System Dynamics 38(6) (2002), 393-414. Swets \& Zeitlinger Pub. Lisse, NE. 

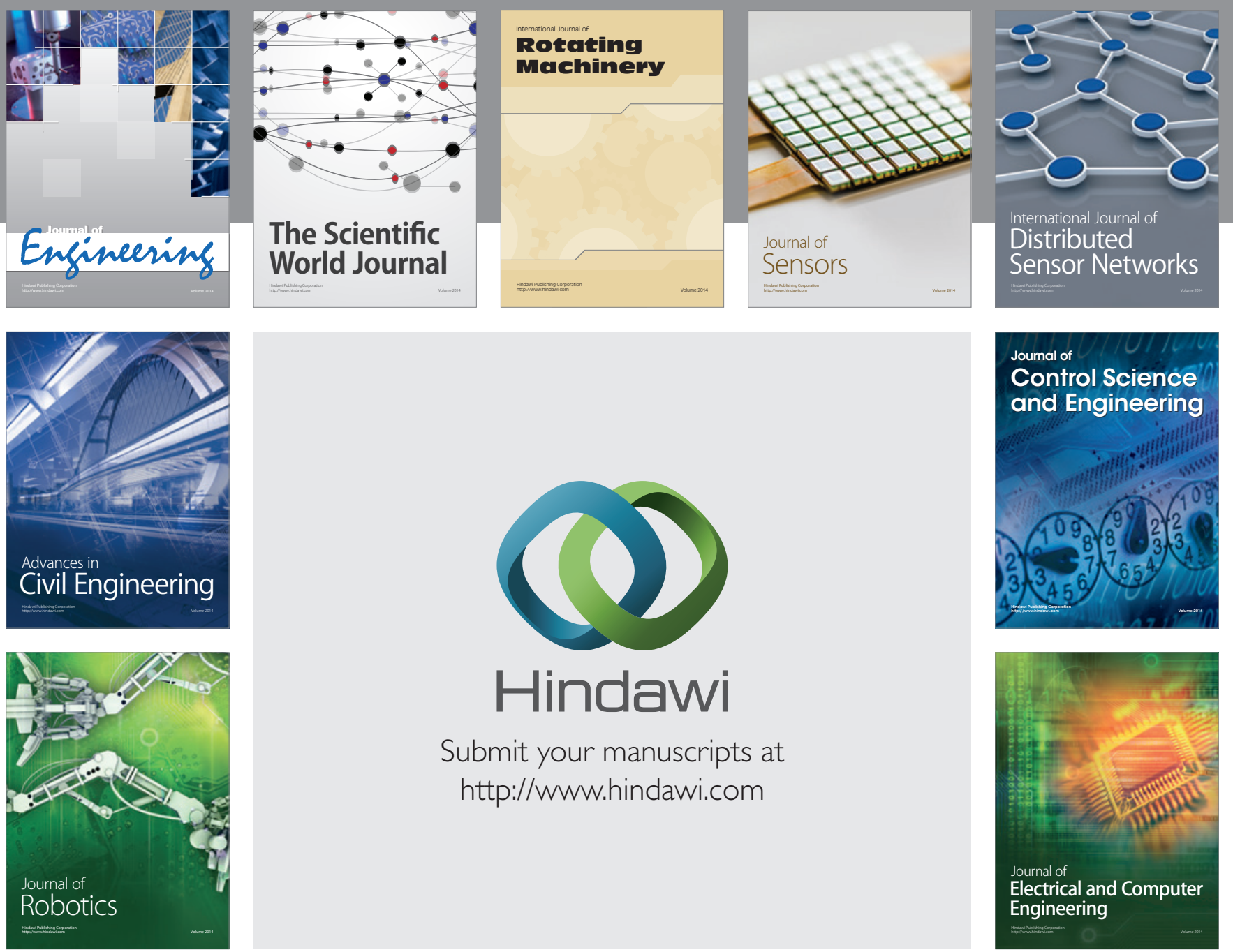

Submit your manuscripts at

http://www.hindawi.com
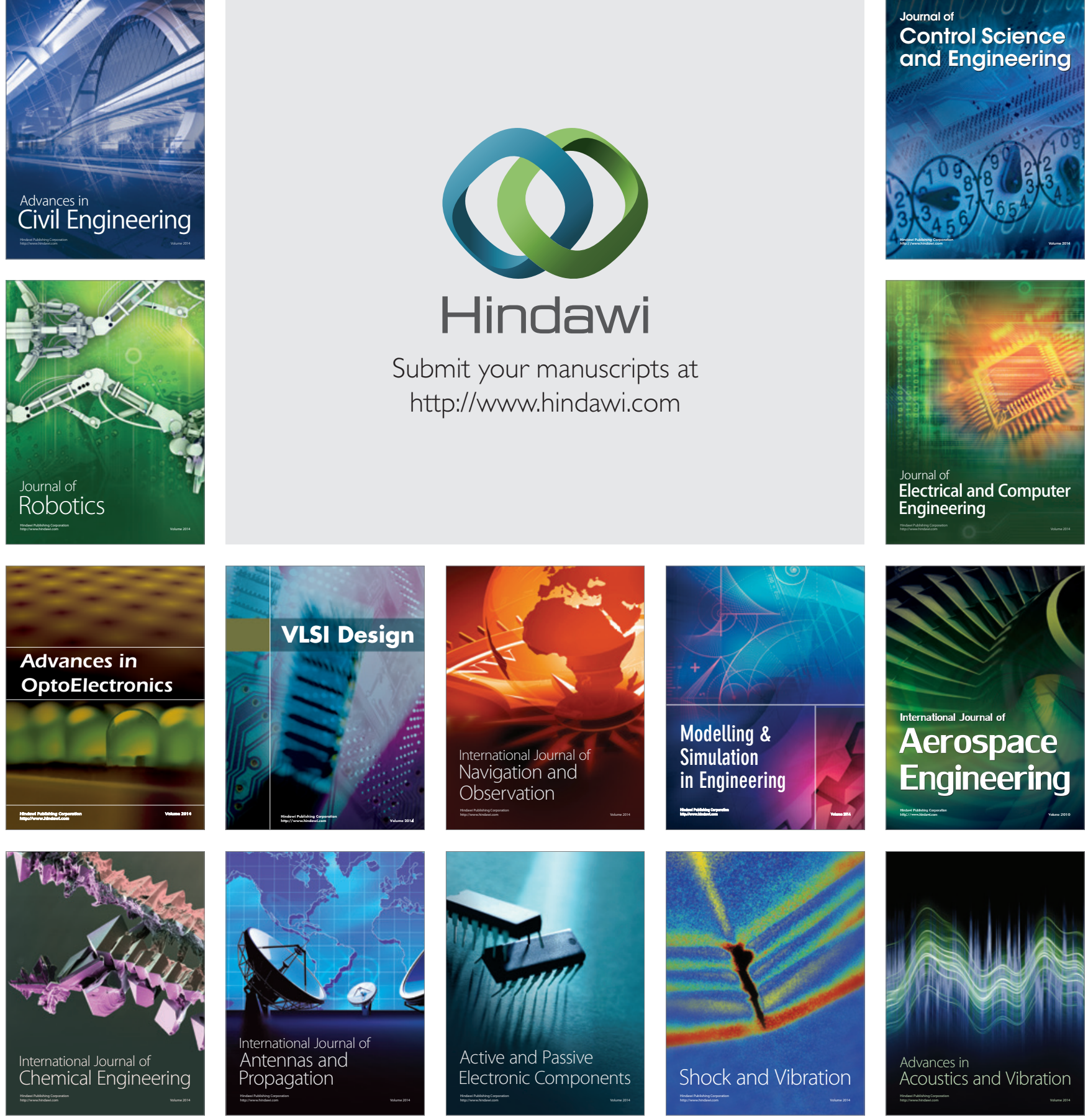